\title{
Detection of Fungal Metabolites from Bakanae Diseased Plants and Their Relationship with Bakanae Disease Symptoms Expression
}

\author{
S. A. J. Quazi ${ }^{1, ~ *, ~ M . ~ S a r i a h ², ~ Z a i n a l ~ A b i d i n ~ B . ~ M . ~ A h m a d ², ~ J . ~ H a w a ~}{ }^{3}$ \\ ${ }^{1}$ Bangladesh Rice Research Institute and Institute of Tropical Agriculture, Universiti Putra Malaysia, Selangor, Malaysia \\ ${ }^{2}$ Department of Plant Protection, Universiti Putra Malaysia, Selangor, Malaysia \\ ${ }^{3}$ Department of Crop Science, Universiti Putra Malaysia, Selangor, Malaysia
}

Email address:

shireenbrri@yahoo.com (S. A. J. Quazi)

${ }^{*}$ Corresponding author

\section{To cite this article:}

S. A. J. Quazi, M. Sariah, Zainal Abidin B. M. Ahmad, J. Hawa. Detection of Fungal Metabolites from Bakanae Diseased Plants and Their Relationship with Bakanae Disease Symptoms Expression. American Journal of Bioscience and Bioengineering.

Vol. 4, No. 6, 2016, pp. 77-89. doi: 10.11648/j.bio.20160406.14

Received: November 28, 2016; Accepted: December 23, 2016; Published: January 17, 2017

\begin{abstract}
Amount of fungal metabolites present in diseased plants and their consequences in bakanae disease development were determined. Metabolites fusaric acid (FA), fumonisin (FB1), moniliformin (MON) and beauvericin (BEA) were isolated and quantified using HPLC analysis from rice plants infected with Fusarium proliferatum. Higher amount of moniliformin was detected from stem part (550 ng/g fresh wt.) as well as from whole plants (112.8 ng/g fresh wt.) in susceptible of MR 211 at disease score level 5. But moniliformin was not detected in inoculated resistant variety BR3. The level of FA was progressed from disease score 1 to disease score 5 that made plants to stunting/ceased growth. Among the four fungal metabolites, MON and FA had found positive relationship with bakanae disease symptoms development. This paper will be helpful for understanding the relationship between fungal metabolites in bakanae disease development in susceptible rice plants. Theknowledge of this research will be also implied on other fungal diseases for which fungi are capable to produce metabolites in infected plants.
\end{abstract}

Keywords: Fusarium Proliferatum, Bakanae, Fungal Metabolites, Symptoms Expression, Susceptible Rice Plants

\section{Introduction}

Bakanae is one of the major diseases in rice growing areas of the world. Although a number of Fusarium(F.) species have been isolated and identified from bakanae diseased plants, F. fujikuroi was found to be prominent in bakanae disease development until 2013. Recently, F. proliferatum has been identified and reported as a causal pathogen of bakanae[1]. Fusarium fujikuroiand $F$. proliferatum both pathogens are similar in terms of morphology and biology and both can produce $\mathrm{GA}_{3}$ [2-5]. In addition, it has been reported that abnormal internode elongation of bakanae diseased plants is due to the higher amounts of gibberellic acids $\left(\mathrm{GA}_{3}\right)$ production by the causal pathogen itself in diseased plants [6-7]. Along with the increased level of $\mathrm{GA}_{3}$ and its interaction with up/down regulation of other phytohormones level such as indole acetic acid and abscisic acid in relation to bakanae disease development has already been established [2].

Besides phytohormones production and/or their levels regulation in diseased plants, $F$. fujikuroi and F. proliferatum both pathogens can produce a wide range of metabolites including fumonisin (FB1), moniliformin (MON), beauvericin (BEA) and fusaric acid (FA). In conjunction with phytohormonal involvement, it was also essential to find out whether, $F$. proliferatum can produce fungal metabolites in bakanae diseased plants or not. The metabolites produced by $F$. fujikuroi and $F$. proliferatum have been identified to have some phytotoxic effects, but their production in bakanae diseased plants in association with bakanae symptoms expression have not been reported [8-11]. 
Plant pathogen infection causes disruption of normal physiological processes by using a variety of biochemical factors in plants. These biochemical factors include cell wall degrading enzymes/PR-proteins, phytohormones, and toxic compounds/metabolites. Although a wide range of fungal toxins have been identified in plant pathogen interactions but the role of these phytotoxins in relation to plant pathogenesis is contentious. On contrary, it has been well established that many mycotoxin-producing fungal species cause plant disease under field conditions [12]. Although the role of metabolites was not clear in bakanae disease development but, it was suspected that the bakanae infection due to $F$. proliferatum might be due to production of metabolites [13]. Moreover, it is obscured whether there is a correlation between the amountsof fungal metabolites produced by $F$. proliferatumwith different bakanae disease symptoms.

Therefore, the aims of this part of the research were to: (1) detectand quantify the fungal metabolites in bakanae diseased plant in comparison with healthy plant; (2) correlate the amount of fungal metabolites with different disease symptoms at different disease scoring levels.

\section{Materials and Methods}

\subsection{Fungal Mycelium Harvest}

Fungal mycelium was harvested from single conidium culture of virulent $F$. proliferatum. Detail procedure was followed by Quazi et al. [2].

\subsection{Inoculation Method and Plant Growth Management}

Inoculation and plant growth management were done as presented by Quazi et al.[2]. In brief,seeds ofsusceptible variety, MR 211 and resistant variety BR3 were surface sterilized with $70 \%$ ethanol, washed with sterilized distilled water and then soaked overnight in sterilized distilled water. The water was drained out and seeds were further soaked in spore suspension $\left(10^{6}\right.$ conidia $\left./ \mathrm{mL}\right)$ of the virulent isolate $F$. proliferatumfor $48 \mathrm{~h}$. The seeds were then planted in steam sterilized soil in trays ( $2 \mathrm{~kg}$ soil/tray) and arranged in a completely randomized design with three replications (60 seeds/replication). Pre-soaked seeds for the control treatment were soaked further in sterile distilled water for $48 \mathrm{~h}$ before sowing. All trays were placed in a glasshouse at room temperature, and watered once daily with a hand sprinkler. Fertilizer comprising of N: P: K (15:15:15) were applied at 4 $\mathrm{g} /$ tray, twice at 15 day intervals.

\subsection{Sampling Procedure}

Disease severity scale from 1 to 5 for Bakanae disease assessment was used as illustratedbyQuaziet al. [1]. But, only three disease score levels (1,3 and 5) were chosen for sampling of metabolites analysis whereas, score $1=$ plant stunted with chlorotic leaves, score 3 = abnormal elongated internodes with chlorotic or brownish leaves, and score $5=$ leaf and stem browning with elongated internodes, fungal masses produced on the infected plant or dead plant. Detail sampling procedure wasas followed for phyhormone analysis and demonstrated by Quazi et al. [2]. Plant samples were collected randomly at 7,14 and 21 days after inoculation(DAI) along with control (healthy) plants. Analysis was carried out on whole plant samples (WP) and from individual plant parts viz. leaves, stems and roots.

\subsection{Extraction, Detection and Quantification of Metabolites}

\subsubsection{Fumonisin (FB1)}

i Chemicals and Reagents: Mobile phase (A) 50\% phosphate buffer mixed with 50\% HPLC grade methanol, and (B) 100\% methanol (HPLC grade, Sigma-Aldrich) was used. Each mobile phase was filtered through $0.2 \mu \mathrm{m}$ nylon membrane (Germany) before use. Phosphate buffer was prepared by dissolving $0.1 \mathrm{M}$ potassium dihydrogen phosphate, anhydrous (SYSTERM, 99.5\%) in ultra pure water (MQ) and $\mathrm{pH}$ of this buffer was adjusted to 7.0 with $0.1 \mathrm{M} \mathrm{NaOH}$ (Sigma-Aldrich, >98.0\%). ACCQ-Fluor $\mathrm{T}^{\mathrm{M}}$ Reagent Kit (Waters, WA, T05 2880, USA) was used following the manufacturer's instructions. Strong anion exchange columns SAX (Supelco, Inc., Bellefonte, PA, 16823-0048) were used for cleanup of sample extracts before derivation.

ii Standard curve preparation: FB1 was purchased from Sigma-Aldrich. A stock solution $(1 \mathrm{mg} / \mathrm{mL})$ was prepared in water (MQ): acetonitrile (HPLC grade) $(1: 1)$ solution. Then a series of concentrations $(0,5,10$, 20 and $50 \mu \mathrm{g} / \mathrm{mL}$ ) were prepared from the stock solution using the same solvent. A standard curve was prepared by injecting $50 \mu \mathrm{L}$ of standard samples derivatized with ACCQ-Fluor Reagent Kit. Finally, a standard curve was prepared using peak area covered for each concentration of FB1 standard and amount of FB1 was determined in micrograms $(\mu \mathrm{g})$.

iii Derivatization procedure:ACCQ-Fluor borate buffer $(60 \mu \mathrm{L})$ was added with a fraction $(20 \mu \mathrm{L})$ of each of the cleaned-up sample extract and standards in $1.5 \mathrm{~mL}$ centrifuge tubes separately. Then $20 \mu \mathrm{L}$ of ACCQFluor reagent were added to each tube. Each reaction mixture was left to stand for $1 \mathrm{~min}$ at room temperature and then heated for $10 \mathrm{~min}$ at $55^{\circ} \mathrm{C}$.

iv Sample extraction procedure:Sample was prepared according to Kovačić et al. [14]with minor modifications. Briefly, One gram ( $1 \mathrm{~g})$ samples of fungal mycelium, whole plant or as different parts including leaves, stems and roots were ground to powder separately in liquid nitrogen using mortar and pestle irrespective of different days (7, 14 and 21) after inoculation and replication wise. Then the ground sample was placed into a $50 \mathrm{~mL}$ polyethylene sample tube and a solution of mixture of $5 \mathrm{~mL}$ methanol (HPLC grade): acetonitrile (HPLC grade): water (MQ) (25: 25:50) was added. The samples were placed in a horizontal shaker and shaken for $30 \mathrm{~min}$ at $200 \mathrm{rpm}$. After that, the extract was filtered to a new sample tube 
using Whatman No. 1 filter paper. Then hexane $(4 \mathrm{~mL})$ was added to partition the samples into two phases. When the two phases were visible, the upper phase was removed using micropipette $(1 \mathrm{~mL})$ and the $\mathrm{pH}$ of the lower phase was adjusted to 8-9 using $\mathrm{NaOH}$ (SigmaAldrich, >98.0\%) (5 M). Chloroform (Fisher chemicals, $99.99 \%$ ) (4 mL) was added to the lower phase, vortexed for mixing, and left aside until separation was formed. The water phase (upper phase) was taken up using a micropipette and loaded onto SAX-SPE column for clean up. Samples from control plants were also prepared following the same procedure.

$\mathrm{v}$ Clean up, chromatographic and quantification procedure:A disposable SAX-SPE column was fitted on the vacuum manifold, Mediwax (R). The column was conditioned by washing with $1 \mathrm{~mL}$ of methanol (HPLC grade): water (MQ) $(3: 1)$. Then the extracted samples were loaded on to the column and washed with $1 \mathrm{~mL}$ of methanol (HPLC grade): water (MQ) (3:1). Again the column was washed with $1 \mathrm{~mL}$ of HPLC grade methanol. Then samples were eluted with $5 \mathrm{~mL}$ acetic acid (Sigma-Aldrich, >99\%): methanol (HPLC grade) (1: 99). The eluted samples were then evaporated to dryness using rotary evaporator at $65^{\circ} \mathrm{C}$. The extracted residue was then dissolved in $200 \mu \mathrm{L}$ methanol (HPLC grade).

The HPLC system equipped with a separation module (Waters e2695) and a Multi $\lambda$ Flurorescence Detector (Waters e2475) was used with excitation at $250 \mathrm{~nm}$ and emission at $395 \mathrm{~nm}$ cutoff filter. The reversed phase column Symmetry (Waters), $4.6 \times 150 \mathrm{~mm}(5 \mu \mathrm{m})$ was used. The gradient program was started at $100 \% \mathrm{~A}$, then ramped to $70 \% \mathrm{~A}$ and $30 \% \mathrm{~B}$ at $11 \mathrm{~min}$ and finally held for $2 \mathrm{~min}$. The total analysis time was $20 \mathrm{~min}$. The LC column was kept at room temperature $\left(30^{\circ} \mathrm{C}\right)$ with a flow rate of $1 \mathrm{~mL} / \mathrm{min}$. Sample solution $(50 \mu \mathrm{L})$ was injected along with a FB1 standard solution. Sample peak was identified by comparing retention time of the reference standard. The quantity of FB1 from samples was determined from the standard curve of the standards and was expressed as $\mu \mathrm{g} / \mathrm{g}$ fresh weight.

\subsubsection{Moniliformin (MON)}

Moniliformin extraction and quantification methods followed after Munimbazi and Bullerman[15]. The procedure is described as follows:

i Solvents and reagents:Acetonitrile (HPLC grade), dichloromethane (HPLC grade), methanol (HPLC grade), deionized ultra pure water, Milli Q (MQ), tetrabutylammonium hydrogen sulfate (SigmaAldrich, >99.0\%), 0.05 Mpotassium dihydrogen phosphate (SYSTERM, 99.5\%) (pH 5.0), $1.1 \mathrm{M}$ sodium dihydrogen phosphate monohydrate (SigmaAldrich, $>99.0 \%$ ), and 0.1 Mortho-phosphoric acid (Sigma-Aldrich, >85\%) were used. Potassium hydroxide (KOH, Sigma-Aldrich, $>85 \%$ ) was used for pH adjustment. ii Mobile phase preparation:Fifty $\mathrm{mL}$ of $40 \%$ terbutylammonium hydrogen sulfate (SigmaAldrich, $>99.0 \%$ ) (w/v) was mixed with $100 \mathrm{~mL}$ of 1.1 $\mathrm{M}$ potassium dihydrogen phosphate (SYSTERM, 99.5\%) to prepare a concentrated ion-pair modifier solution. Then the concentrated solution was diluted by mixing $10 \mathrm{~mL}$ of concentrated ion-pair modifiers with water (MQ) to make a final volume of 1 liter. The $\mathrm{pH}$ of the diluted solution was adjusted to 6.5 with $5 \mathrm{~N}$ $\mathrm{KOH}(\mathrm{KOH}$, Sigma-Aldrich, $>85 \%)$. After that, $8 \%$ acetonitrile (HPLC grade) solution was prepared using the prepared diluted ion-pair modifiers and then filtered through $47 \mathrm{~mm} \times 0.20 \mu \mathrm{m}$ nylon membrane (Whatman, GmbH, Germany).

iii Preparation of working standard solution and standard curve:Pure sodium salt of moniliformin (Sigma Chemical Co. St. Luis, Mo, USA) was dissolved (1000 $\mu \mathrm{g} / \mathrm{mL}$ ) in $0.05 \mathrm{M}$ sodium hydrogen phosphate monohydrate, $\mathrm{pH}$ 5.0. An intermediate solution of moniliformin standard $(1 \mu \mathrm{g} / \mathrm{mL})$ was prepared. Different concentrations of moniliformin $(100,200$, 300,400 and $500 \mathrm{ng} / \mathrm{mL}$ ) was prepared from the intermediate solution of the standard using the same solvent. The solutions were stored at $4{ }^{\circ} \mathrm{C}$ until analysis in HPLC. A standard curve was prepared using peak area covered by injecting $50 \mu \mathrm{L}$ of MON standard at different concentrations as described before. Amount of MON was determined in nanograms (ng).

iv Sample extraction procedure:Sample used for extraction was done as similar as FB1 and ground. Then the ground sample was taken into a $50 \mathrm{~mL}$ polyethylene sample tube. Five (5) $\mathrm{mL}$ of $1 \%$ terbutylammonium hydrogen sulfate (TBAHS, SigmaAldrich, $>99.0 \%$ ) was prepared in MQ water and was added to the ground sample. The samples were placed in a horizontal shaker and shaken for $30 \mathrm{~min}$ at 200 rpm. After that, the extract was filtered to a new sample tube using Whatman No. 1 filter paper. Then 5 $\mathrm{mL}$ of $1 \%$ terbutylammonium hydrogen sulfate was added to individual sample tubes containing sample residues and shaken as before. Then the second extract was filtered and the two extracts of each sample were combined. After that the combined extracts $(10 \mathrm{~mL})$ were transferred into a pear shaped flask and $10 \mathrm{~mL}$ of dichloromethane (HPLC grade) was added and mixed gently without vigorous shaking. Then waited until the two phases were separated and the upper phase was discarded using a micropipette $(1 \mathrm{~mL})$. The separation step was repeated using another $10 \mathrm{~mL}$ dichloromethane (HPLC grade) and two dichloromethane extracts were combined. The dichloromethane extracts were evaporated to dryness using rotary evaporator at $55^{\circ} \mathrm{C}$. The extract residue was then dissolved in $1 \mathrm{~mL}$ of MQ water and was loaded onto the Solid Phase Extraction (SPE) column. Samples from control plants were also prepared following the same procedure. 
v Extract cleanup procedure: Method was followed by Munimbazi and Bullerman [15].

vi Chromatographic and quantification procedure

A PDA detector (Waters, 2998) with wave length $227 \mathrm{~nm}$ was used. Column Phenomenex, Torrance CA (150 X 4.6 $\mathrm{mm}$ id, $5 \mu \mathrm{m}$ ) was used in Waters separation module (e2695). Mobile phase was used isocratically at a flow rate of 1 $\mathrm{mL} / \mathrm{min}$. Sample solution $(50 \mu \mathrm{L})$ was injected along with a moniliformin standard solution. Sample peak was identified by comparing retention time of the reference standard. The quantity of moniliformin from samples was determined from the standard curve and was expressed as ng/g fresh weight.

\subsubsection{Beauvericin (BEA)}

i. Chemicals and reagents:Acetonitrile (HPLC grade), dichloromethane (HPLC grade), grade), hexane (HPLC grade), ammonium acetate (Sigma-Aldrich, >99.0\%), and deionized ultra pure water (MQ) were used.

ii. Preparation of working standard solution and standard curve preparation: BEA was purchased from Sigma Chemical Co. St. Luis, Mo, USA. The metabolite was dissolved $(1000 \mu \mathrm{g} / \mathrm{mL}$ ) in methanol (HPLC grade) as stock solution. Then different concentrations of BEA $(0,5,10,200$ and $50 \mu \mathrm{g} / \mathrm{mL})$ was prepared from the stock solution of the standard using the same solvent. The solutions were stored at $-20^{\circ} \mathrm{C}$ until analysis in HPLC. A standard curve was prepared using peak area covered by injecting $50 \mu \mathrm{L}$ of BEA standard at different concentrations as described before. Amount of BEA was determined in micrograms $(\mu \mathrm{g})$.

iii. Sample extraction procedure:Sample was prepared according to Kostecki et al.[16]with some modifications for BEA extraction. Sample was taken for extraction as similar as of FB1and ground to powder separately and replication wise. Then the ground sample was taken into a $50 \mathrm{~mL}$ polyethylene sample tube. After that, $5 \mathrm{~mL}$ of extraction solvent (acetonitrile [HPLC grade]: methanol [HPLC grade]: water $[\mathrm{MQ}]=16: 3: 1)$ was added and shaken in a horizontal shaker for $2 \mathrm{~h}$ at $200 \mathrm{rpm}$. The extracts were then filtered to a new sample tube using Whatman No. 1 filter paper. After that $5 \mathrm{~mL}$ hexane (HPLC grade) was added to the extracts in the tube for partitioning the samples into two phases. When the two phases were visible the upper phase was removed using micropipette $(1 \mathrm{~mL})$ and the bottom phase was evaporated to dryness using rotarory evaporator at $60^{\circ} \mathrm{C}$. The extract residue was dissolved in $10 \mathrm{~mL}$ Methanol (HPLC grade): water (MQ) (1: 1). After that $5 \mathrm{~mL}$ dichloromethane (HPLC grade) was added to the dissolved extract residue in tube for further separation. The upper phase was removed using micropipette (1 $\mathrm{mL}$ ) and bottom phase (dichloromethane) was collected. This separation step was repeated twice and the two collected phases were pooled and evaporated to dryness using rotary evaporator at $60^{\circ} \mathrm{C}$. The extract residue was dissolved in $200 \mu \mathrm{L}$ methanol (HPLC grade) and was stored at $-20^{\circ} \mathrm{C}$ until LC analysis. Samples from control plants were also prepared following the same procedure. The samples were analyzed without cleanup procedure.

iv. Chromatographic and quantification procedure:Column Phenomenex, Torrance CA (150 X $4.6 \mathrm{~mm}$ id, $5 \mu \mathrm{m}$ ) was used in a separation module (Waters e2695) for HPLC analysis. A PDA detector (Waters 2998) with wave length $205 \mathrm{~nm}$ was used. Mobile phase ammonium acetate (HPLC grade) (A), methanol (HPLC grade) (B) and acetonitrile (HPLC grade) (C) was used isocratically in the ratio of 45: 45: 10. Flow rate was $0.6 \mathrm{~mL} / \mathrm{min}$. Sample solution $(50$ $\mu \mathrm{L})$ was injected along with a BEA standard solution. Sample peak was identified by comparing retention time of the reference standard. The quantity of BEA from samples was determined from the standard curve and was expressed as $\mu \mathrm{g} / \mathrm{g}$ fresh weight.

\subsubsection{Fusaric Acid (FA)}

$\mathrm{i}$ Chemicals and reagents:Ethyl acetate (HPLC grade), methanol (HPLC grade) and phosphoric acid (HPLC grade), ethyl acetate (HPLC grade) and deionized ultra pure water (MQ) were used. Hydrochloric acid (Sigma-Aldrich, $>37 \%$ ) was used for $\mathrm{pH}$ adjustment.

ii Preparation of working standard solution and standard curve preparation:FA acid was purchased from Sigma Chemical Co. St. Luis, Mo, USA, as powdered form. The acid was dissolved $(1000 \mu \mathrm{g} / \mathrm{mL})$ in methanol (HPLC grade) and used as stock solution. Then different concentrations of FA $(0,5,10,20$ and 50 $\mu \mathrm{g} / \mathrm{mL}$ ) were prepared from the stock solution of the standard using the same solvent. The solutions were stored at $-20^{\circ} \mathrm{C}$ until analysis in HPLC. A standard curve was prepared by injecting $50 \mu \mathrm{L}$ of FA standard at different concentrations as described before. Amount of FA was determined in micrograms $(\mu \mathrm{g})$.

iii Sample extraction procedure: Sample was prepared for FA extraction according to Eged [17]. Sample of whole plant $(1 \mathrm{~g})$, or different parts (1g) or fungal mycelium (1g) were chosen as similar as other metabolites extract prepared before and ground. Then the ground sample was taken into a $50 \mathrm{~mL}$ polyethylene sample tube and $30 \mathrm{~mL}$ MQ water was added to the sample in the tube. The samples were placed in a horizontal shaker and were shaken for $30 \mathrm{~min}$ at $200 \mathrm{rpm}$, and then placed in a refrigerator for $1 \mathrm{~h}$. After that, the extract was filtered to a new sample tube using Whatman No. 1 filter paper. The solution was made acidic by adding $1 \mathrm{~mL}$ of 2 N HCL (Sigma-Aldrich, $>37 \%)(\mathrm{pH} 3.9-4.0)$. Then $30 \mathrm{~mL}$ of ethyl acetate (HPLC grade) was added to the sample tube. This step was repeated 2 times. The ethyl acetate extracts were pooled and evaporated to dryness at $50^{\circ} \mathrm{C}$ using rotary evaporator. The sample residue was dissolved in $1 \mathrm{~mL}$ of $80 \%$ ethanol (SigmaAldrich, $>99.5 \%$ ). After that, the sample extracts were concentrated to $400 \mu \mathrm{L}$ using nitrogen gas flow and 
stored in $-20^{\circ} \mathrm{C}$ until LC analysis. Samples from control plants were also prepared following the same procedure. The samples were analyzed without cleanup procedure.

iv Chromatographic and quantification procedure:Column Symmetry (Waters) (150 X $4.6 \mathrm{~mm}$ id, $5 \mu \mathrm{m}$ ) was used in a separation module (Waters e2695) for HPLC analysis. A PDA detector (Waters 2998) with wave length $268 \mathrm{~nm}$ was used. Mobile phase was methanol (Sigma-Aldrich, HPLC grade) (A) and $2 \% \mathrm{H}_{3} \mathrm{PO}_{4}$ (Sigma-Aldrich $\left.>85 \%\right)(\mathrm{B})$, and was used isocratically in the ratio of $70 \% \mathrm{~A}$ and $30 \% \mathrm{~B}$. Flow rate was $0.6 \mathrm{~mL} / \mathrm{min}$. The column temperature was maintained at $25^{\circ} \mathrm{C}$. Sample solution $(50 \mu \mathrm{L})$ was injected along with a FA standard solution. Sample peak was identified by comparing retention time of the reference standard and the quantity of FA from samples was determined from the standard curve and expressed as $\mu \mathrm{g} / \mathrm{g}$ fresh weight.

v Metabolites analysis from samples: Chromatographic procedures and mobile phase were used as described before. All the extracted metabolites from samples were filtered through Whatman $0.2 \mu \mathrm{m}$ membrane (Millipore) (Germany) using a filtration syringe system. Fungal metabolites (FB1, MON, BEA and FA) from samples were quantified irrespective of peak area of samples and calculation was carried out irrespective of metabolite standard curve by comparison of retention time and UV absorbance at an optimum point of the standard. All concentrations were reported on per unit weight basis and each sample was analysed in triplicate. Sample peak was identified by comparing retention time of the reference standard and results of each metabolite was analysed and quantified as $\mu \mathrm{g} / \mathrm{g}$ fresh wt. of samples. Means of metabolites $(\mu \mathrm{g} / \mathrm{g}$ fresh wt.) obtained from each disease score level (disease score 1, 3 and 5) and control sample irrespective of disease score level was compared using SAS 9.2 software.

\subsection{Relationship Study of Fungal Metabolites Isolated from Bakanae Diseased Plants}

Regression analyses were done to find out any relationship between the amount of detection levels of fungal metabolites (FB1, MON, BEA and FA) and disease symptoms at different days after inoculation in susceptible variety.

\section{Results}

\subsection{Detection and Quantification of FB1, MON, BEA and FA}

The HPLC responses (peak areas) of FB1, MON, BEA and FA were observed to have highly positive correlations with the respective standard concentrations. The retention times of FB1, MON, BEA and FA were detected from the standard as well as from samples at 8.9, 5.5, 4.08 and $3.5 \mathrm{~min}$, respectively (Figure 1-4).
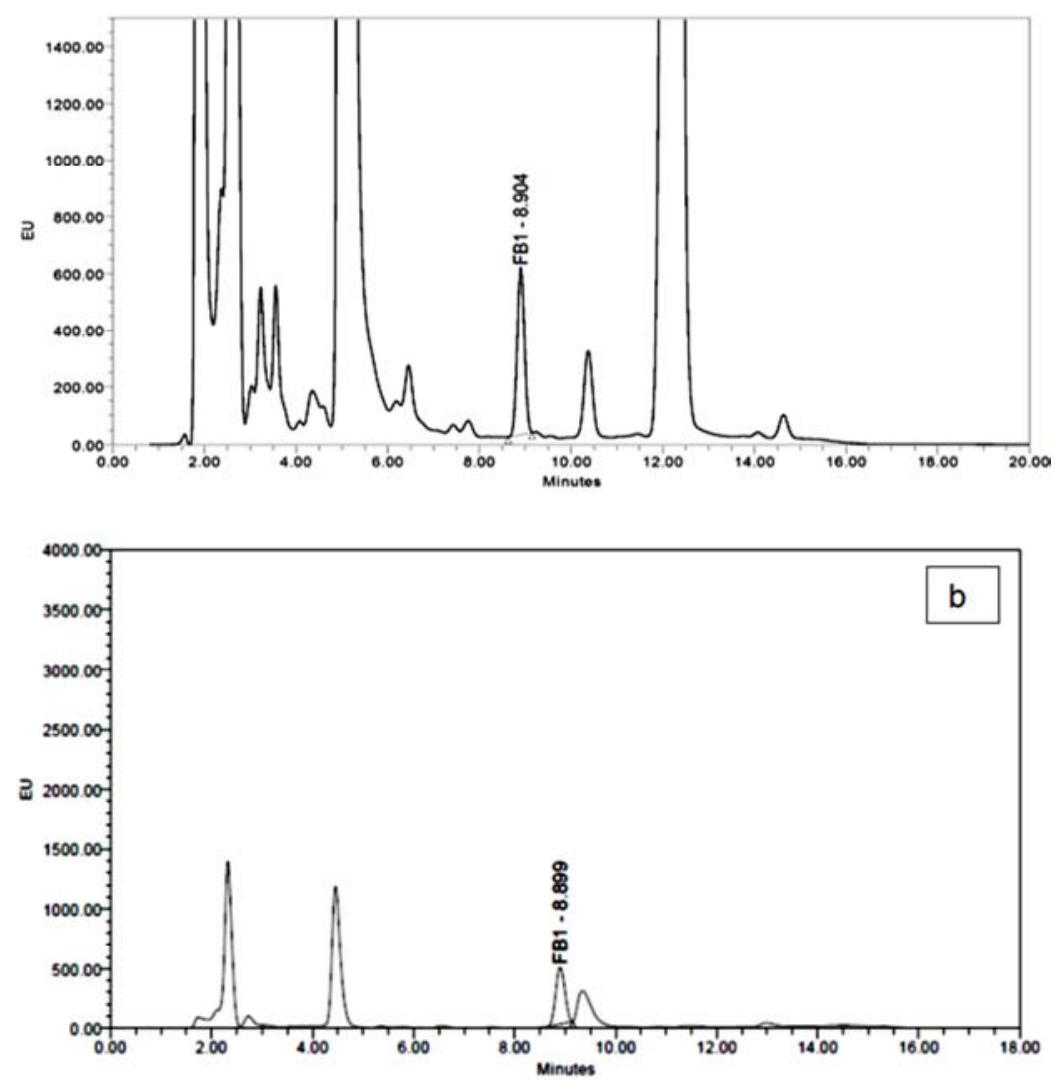

Figure 1. Chromatographs of fumonisin (FB1) peaks with retention time for the sample (a) and standard (b). 

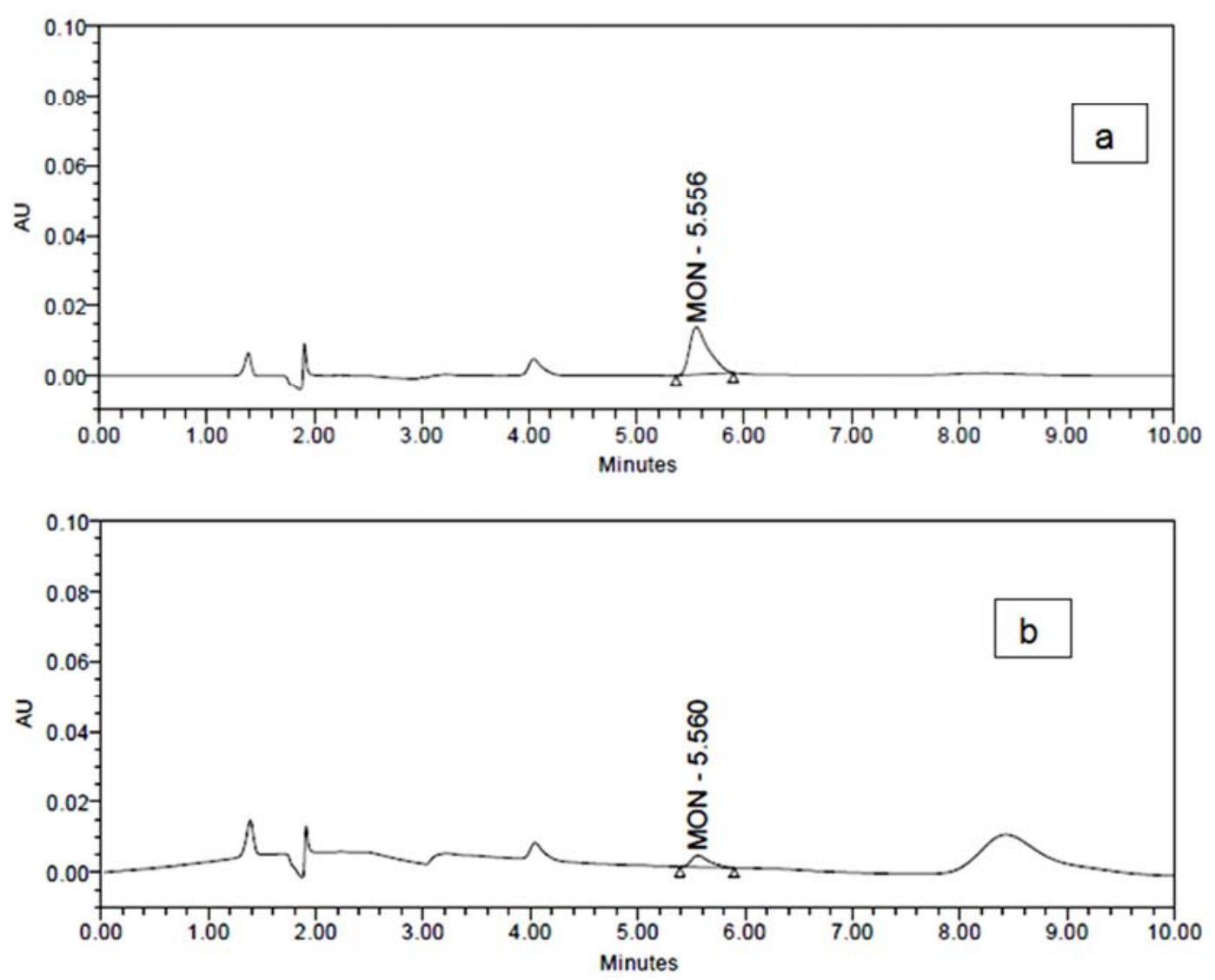

Figure 2. Chromatographs of moniliformin (MON) peaks with retention time for the sample (a) and the standard (b).
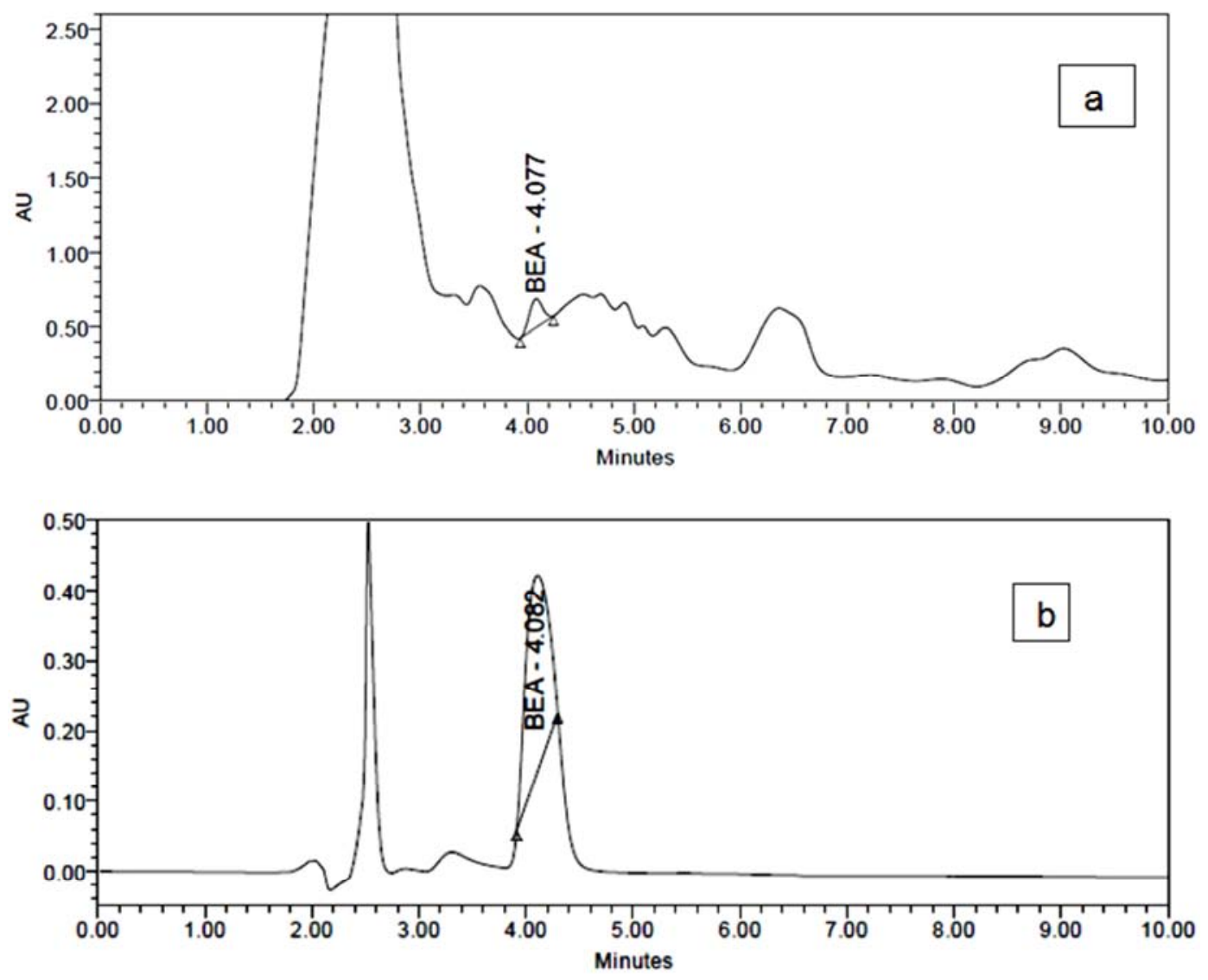

Figure 3. Chromatographs of beauvericin (BEA) peaks with retention time for the sample (a) and the standard (b). 

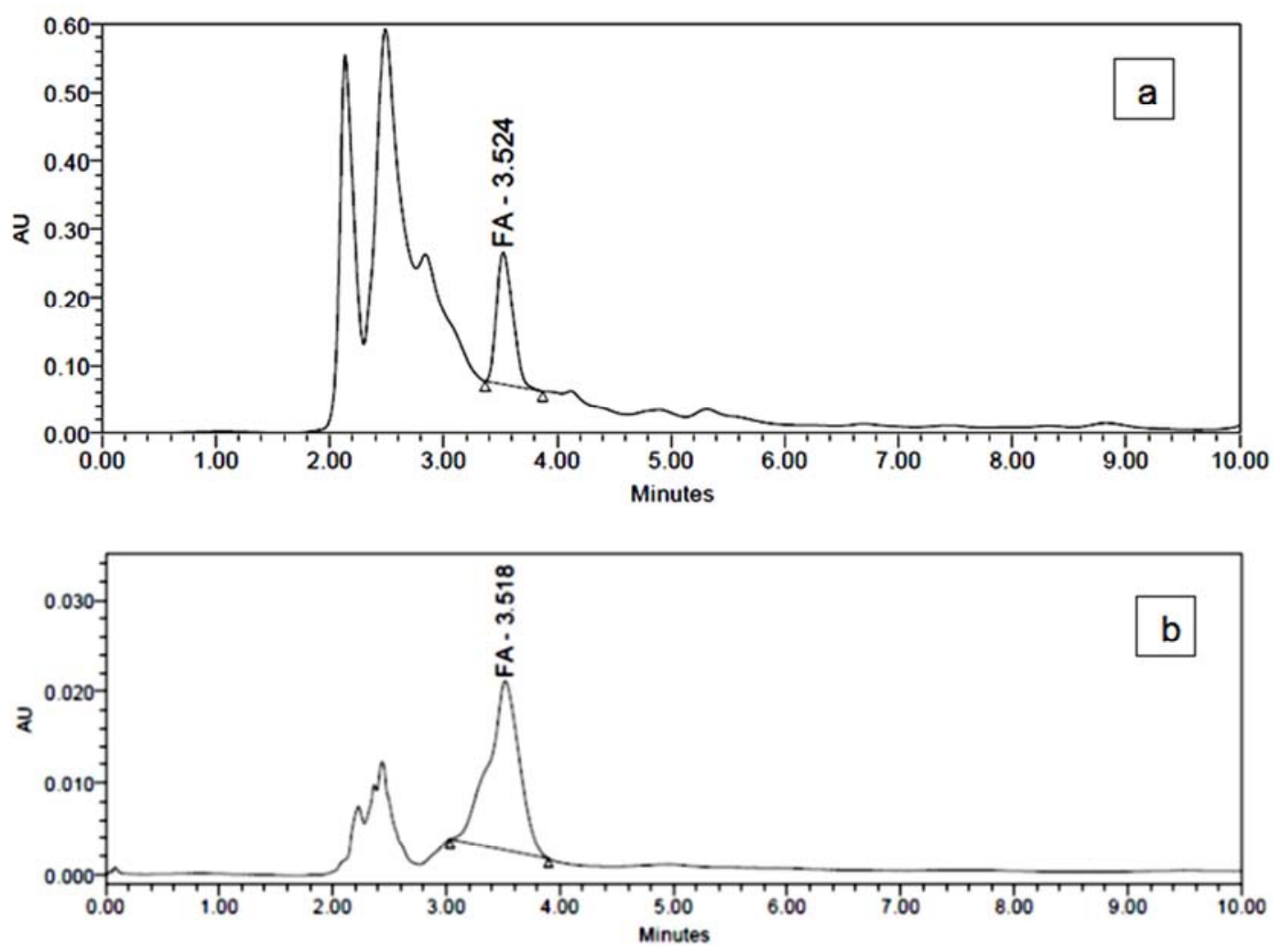

Figure 4. Chromatographs of fusaric acid (FA) peaks with retention time for the sample (a) and the standard (b).

Fumonisin was quantified from fungal mycelium (0.14 $\mu \mathrm{g} / \mathrm{g}$ ) and from infected plant samplesat three disease score levels irrespective of three DAI in susceptible variety MR 211. On the other hand, it was detected at 14 DAI and 21 DAI in whole plant analysis from the resistant variety BR3 (Figure $5 \mathrm{a}$ and $5 \mathrm{~b}$ ). Although a lower amount of FB1 was detected in the fungal mycelium, increasing amounts were detected in infected plants of MR 211 from 7 DAI to 21 DAI. In infected plants of MR 211, FB1 was mainly detected from root tissues at all three DAI (disease score 1, 3 and 5) and a lower amount was detected from stem tissues $(0.21 \mu \mathrm{g} / \mathrm{g})$ at 21 DAI (Table 1). In resistant variety BR3, FB1 was detected from all three parts of infected plants but higher amounts were obtained from leaf and stem tissues compared to root tissues (Table 2). Even though a higher concentration of FB1 was detected from leaves $(6.37 \mu \mathrm{g} / \mathrm{g})$ and from stems (3.99 $\mu \mathrm{g} / \mathrm{g}$ ) of resistant variety BR3 at 21 DAI, but did not contribute to the development of bakanae symptoms in infected plants. FB1 was not detected from control plant samples either in whole plant analysis or from different parts.
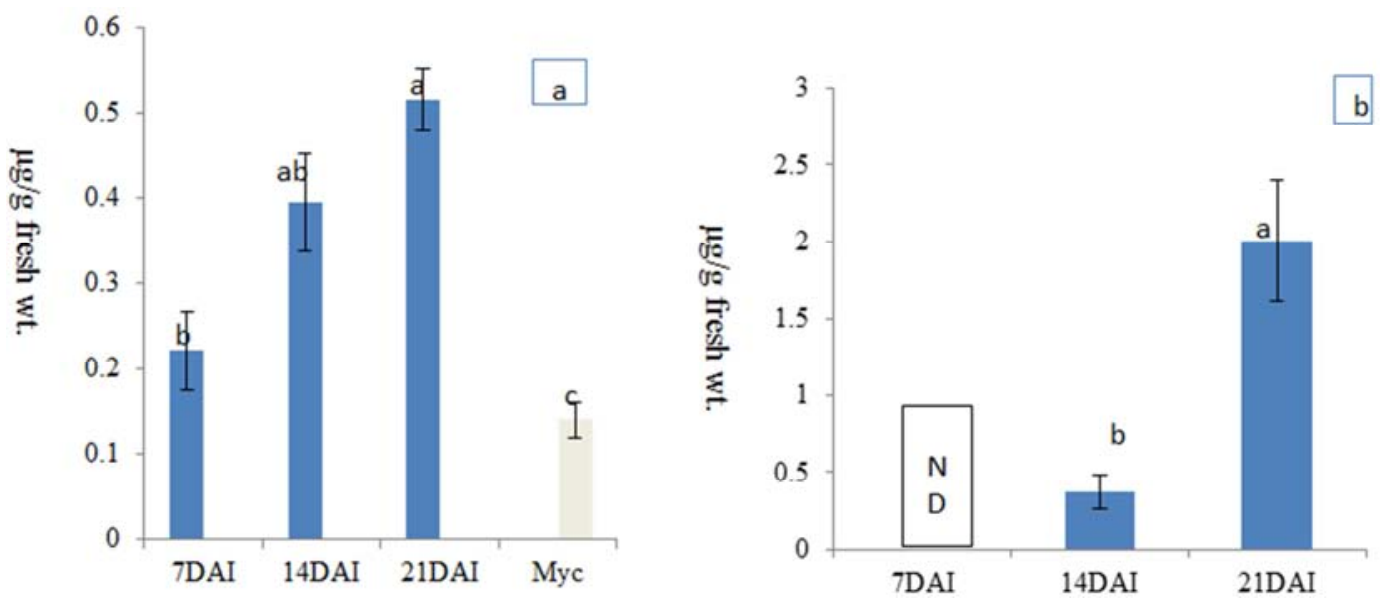

Figure 5. Fumonisin (FB1) quantification in whole plant sample analysis of susceptible variety MR 211 along with fungal mycelium (a) and in resistant variety BR3 (b) infected with Fusarium proliferatum at different disease score levels(Values are means of 3 replications with vertical bars representing standard error). ${ }^{*} N D=$ not detected, $M y c=$ mycelium.

Moniliformin was detected from fungal mycelium (32.41 $\mathrm{ng} / \mathrm{g}$ ) and from infected susceptible variety MR 211 in whole plant sample analysis at $14 \mathrm{DAI}(83.67 \mathrm{ng} / \mathrm{g})$ and at $21 \mathrm{DAI}$ $(112.81 \mathrm{ng} / \mathrm{g}$ ) (Figure 6). Higher amount of MON was 
detected in the stems compared to leaf tissues at 21 DAI (disease score 5) (Table 1). Moniliformin was not detected either from whole plant samples or from individual part of infected resistant variety BR3. Therefore, it was clearly understood that MON possess either pressure or stress on disease development and symptoms expression in the susceptible variety. In addition, resistant plants of BR3 might have the capability to hinder MON production in plants infected with $F$. prolifetratum and thereby showing no symptom. MON was not detected from control plant samples of both varieties either in whole plant analysis or from different plant parts.

Table 1. Metabolites quantified from different parts of plants of susceptible variety MR 211 infected with Fusarium proliferatum at different DAI corresponding to different disease score levels.

\begin{tabular}{|c|c|c|c|c|c|c|}
\hline \multirow{2}{*}{ Parts } & \multicolumn{3}{|c|}{${ }^{*} \mathrm{FB} 1(\mu \mathrm{g} / \mathrm{g}$ fresh wt. $\pm \mathrm{sd})$} & \multicolumn{3}{|c|}{$\operatorname{MON}(n g / g$ fresh wt. \pm sd $)$} \\
\hline & 7 DAI (S-1)** & 14 DAI (S-3) & 21 DAI (S-5) & 7 DAI (S-1) & 14 DAI (S-3) & 21 DAI(S-5) \\
\hline leaf & ND & ND & ND & ND & ND & $328.75 \pm 33.38$ \\
\hline stem & ND & $0.21 \pm 0.84$ & ND & ND & $322 \pm 98.70$ & $550.29 \pm 169.37$ \\
\hline root & $1.30 \pm 0.01$ & $0.24 \pm 0.24$ & $1.54 \pm 0.35$ & ND & ND & ND \\
\hline
\end{tabular}

Table 1. Continue.

\begin{tabular}{llllll}
\hline \multirow{2}{*}{ Parts } & \multicolumn{3}{c}{ BEA $(\boldsymbol{\mu g} / \mathbf{g}$ fresh wt.. \pm sd $)$} & \multicolumn{3}{l}{ FA $(\boldsymbol{\mu g} / \mathbf{g}$ fresh wt.. \pm sd) } \\
\cline { 2 - 7 } & 7 DAI(S-1) & 14 DAI(S-3) & 21 DAI(S-5) & 7 DAI (S-1) & 14DAI (S-3) \\
\hline leaf & $285.11 \pm 61.0$ & $362.33 \pm 56.55$ & $60.62 \pm 13.82$ & $2.16 \pm 0.32$ & $23.19 \pm 6.70$ \\
stem & $84.08 \pm 41.25$ & $14.78 \pm 0.11$ & $13.92 \pm 4.20$ & $108.88 \pm 2.05$ & $13.52 \pm 3.45$ \\
root & $210.82 \pm 0.20$ & $61.80 \pm 25.18$ & $16.13 \pm 2.04$ & $32.90 \pm 9.82$ & $109.99 \pm 36.33$ \\
\hline
\end{tabular}

*FB1=Fumonisin, MON=Moniliformin, BEA= Beauvericin, $\mathrm{FA}=$ Fusaric acid,.DAI= day after inoculation,

$* * \mathrm{~S}-1=$ score 1,7 days after inoculation; $\mathrm{S}-3=$ score 3,14 days after inoculation; $\mathrm{S}-5=$ score 5,21 days after inoculation; (Values are means of 3 replications with \pm standard error). $* \mathrm{ND}=$ not detected. $\mathrm{sd}=$ standard deviation

Table 2. Metabolites quantified from different parts of plants of resistant variety BR3 infected with Fusarium proliferatum at different DAI corresponding to different disease score levels.

\begin{tabular}{|c|c|c|c|c|c|c|c|c|c|c|c|c|}
\hline \multirow{2}{*}{ Parts } & \multicolumn{3}{|c|}{${ }^{*}$ FB1 $(\mu \mathrm{g} / \mathrm{g}$ fresh wt... sd) } & \multicolumn{3}{|c|}{ MON (ng/g fresh wt. \pm sd) } & \multicolumn{2}{|c|}{ BEA $(\mu \mathrm{g} / \mathrm{g}$ fresh wt.. $\pm \mathrm{sd})$} & \multicolumn{4}{|c|}{ FA $(\mu \mathrm{g} / \mathrm{g}$ fresh wt.. $\pm \mathrm{sd})$} \\
\hline & 7 DAI & 14DAI & 21 DAI & 7 DAI & 14 DAI & 21 DAI & 7 DAI & 14 DAI & 21 DAI & 7 DAI & 14 DAI & 21 DAI \\
\hline leaf & ND & $1.26 \pm 0.42$ & 6.37 & ND & ND & ND & $532.06 \pm 0.1$ & $29.57 \pm 0.00$ & $161.55 \pm 3.06$ & ND & ND & ND \\
\hline stem & ND & ND & 3.99 & ND & ND & ND & $282.05 \pm 6.20$ & ND & $192.44 \pm 1.87$ & ND & ND & ND \\
\hline root & ND & $1.85 \pm 0.69$ & 0.97 & ND & ND & ND & $465.30 \pm 150.35$ & $542.85 \pm 81.76$ & $12.09 \pm 0.24$ & ND & ND & ND \\
\hline
\end{tabular}

*FB1=Fumonisin, MON=Moniliformin, BEA= Beauvericin, FA= Fusaric acid. DAI $=$ day after inoculation, (Values are means of 3 replications with \pm standard error). $* \mathrm{ND}=$ not detected.sd $=$ standard deviation

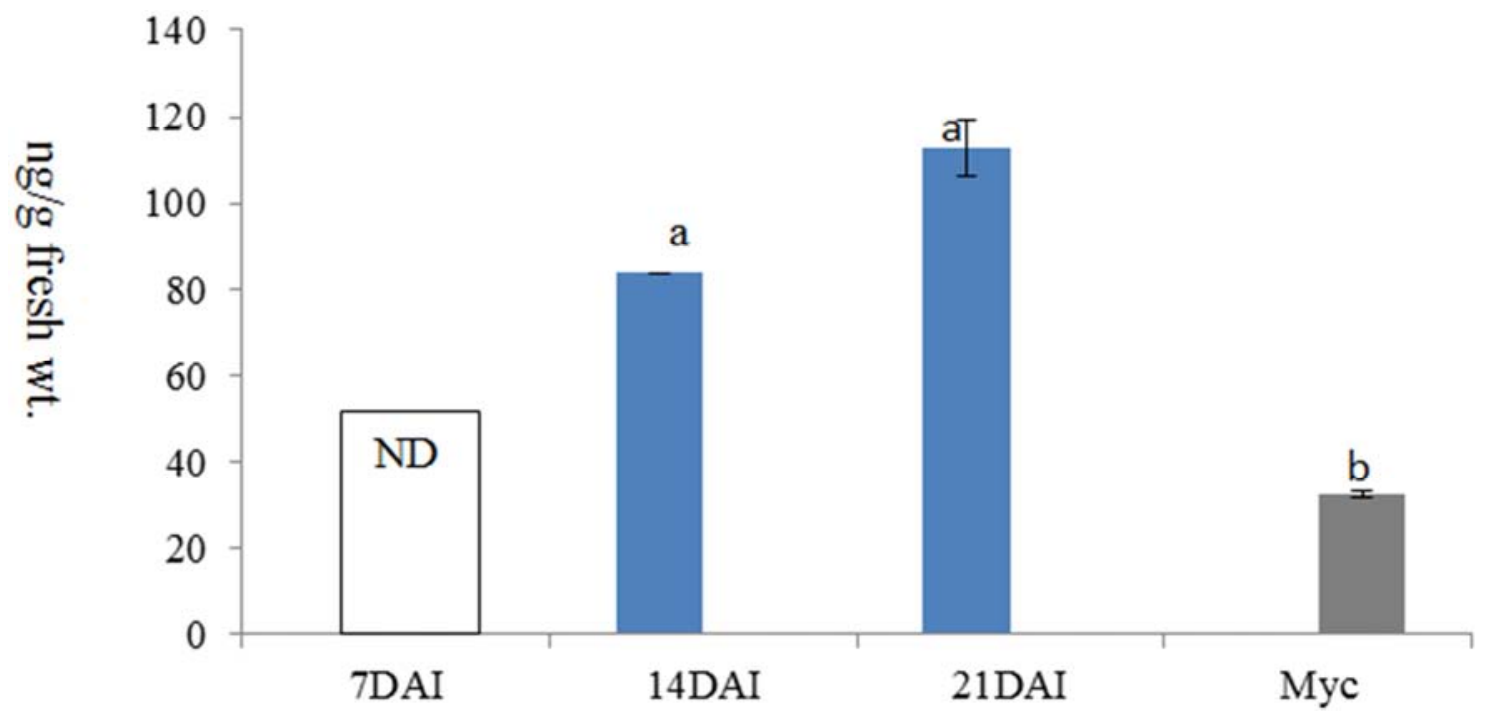

Figure 6. Quantification of MON in whole plant sample analysis of susceptible variety MR 211 infected with Fusarium proliferatum along with fungal mycelium [(Values are means of 3 replications with vertical bars representing standard error). $* N D=$ not detected, Myc $=$ mycelium.

Beauvericin was found at an increasing amount in susceptible variety MR 211 and at decreasing amount in resistant variety from 7 DAI to $21 \mathrm{DAI}$ (disease scores 1 to 5) in whole plant sample analysis (Figure $7 \mathrm{a}$ and $3 \mathrm{~b}$ ). BEA was not detected in fungal mycelium. A significant amount of BEA was detected from different parts at 7DAI for both infected susceptible and resistant varieties (Table 1 and 2). Leaf and root tissues were found to be important domains for 
BEA accumulation compared to stem tissues of both varieties. Although a significant amount of BEA was detected from resistant variety BR3, yet it was found to be involved in disease symptoms expression (i.e. no symptom related to bakanae disease was observed in infected BR3 plants).

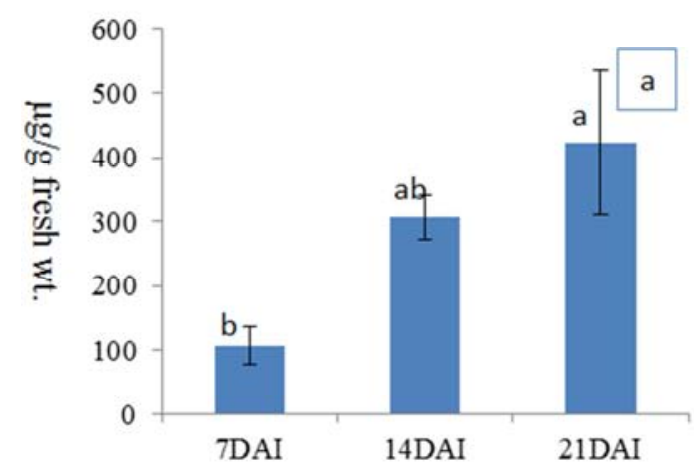

Therefore, it was assumed that BEA has no role in bakanae disease development. BEA was not detected from control plant samples either in whole plant sample or in plant parts analyzed.

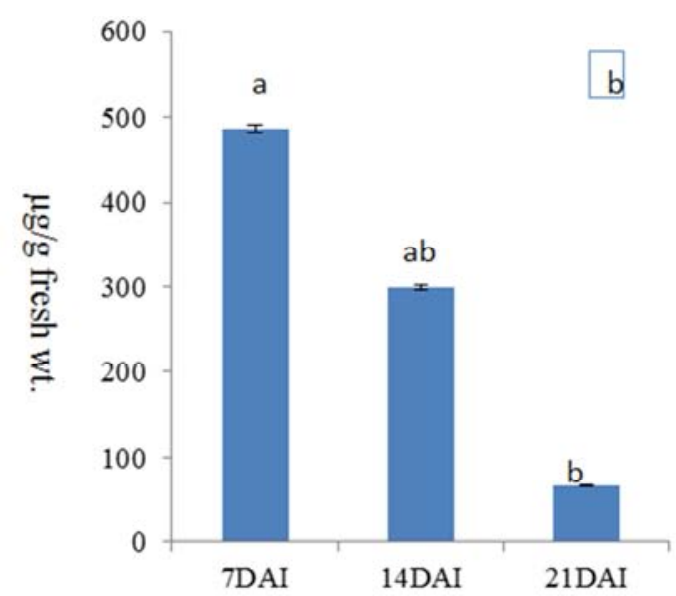

Figure 7. Beauvericin (BEA) quantification in whole plant sample analysis at different disease score levels from susceptible variety MR 211 (a) andfrom resistant variety BR3 (b) infected with Fusarium proliferatum. (Values are means of 3 replications with vertical bar representing standard error).

Fusaric acid was detected in fungal mycelium $(2233 \mu \mathrm{g} / \mathrm{g})$ and in infected susceptible variety MR 211 at all sampling dates irrespective of three disease score levels (disease score 1, 3 and 5) in whole plant analysis. Higher amounts of FA were observed in infected plants at 7DAI (disease score $1=354.41 \mu \mathrm{g} / \mathrm{g}$ ) and $21 \mathrm{DAI}$ (disease score $5=372.38 \mu \mathrm{g} / \mathrm{g}$ ) in whole plant sample analysis (Figure 8). The highest amount was observed in infected leaves $(445.11 \mu \mathrm{g} / \mathrm{g})$ followed by stem tissues $(378.62 \mu \mathrm{g} / \mathrm{g}$ ) at $21 \mathrm{DAI}$ (disease score 5) (Table 1). Although higher amounts of FA production was observed in fungal mycelium of $F$. proliferatum yet the pathogen was not found to have any influence to produce FA in infected resistant variety BR3. Therefore, no detection of FA in BR3 might be due to the capability of this variety to prevent the production of FA in infected plants and resulting in no disease development/ symptom expression. As FA was detected at higher amounts from all parts in infected MR 211, therefore it was assumed that FA has capacity to involve in disease symptoms expression in the susceptible variety. FA was not detected from control plant samples either in whole plant sample or in plant parts analyzed.

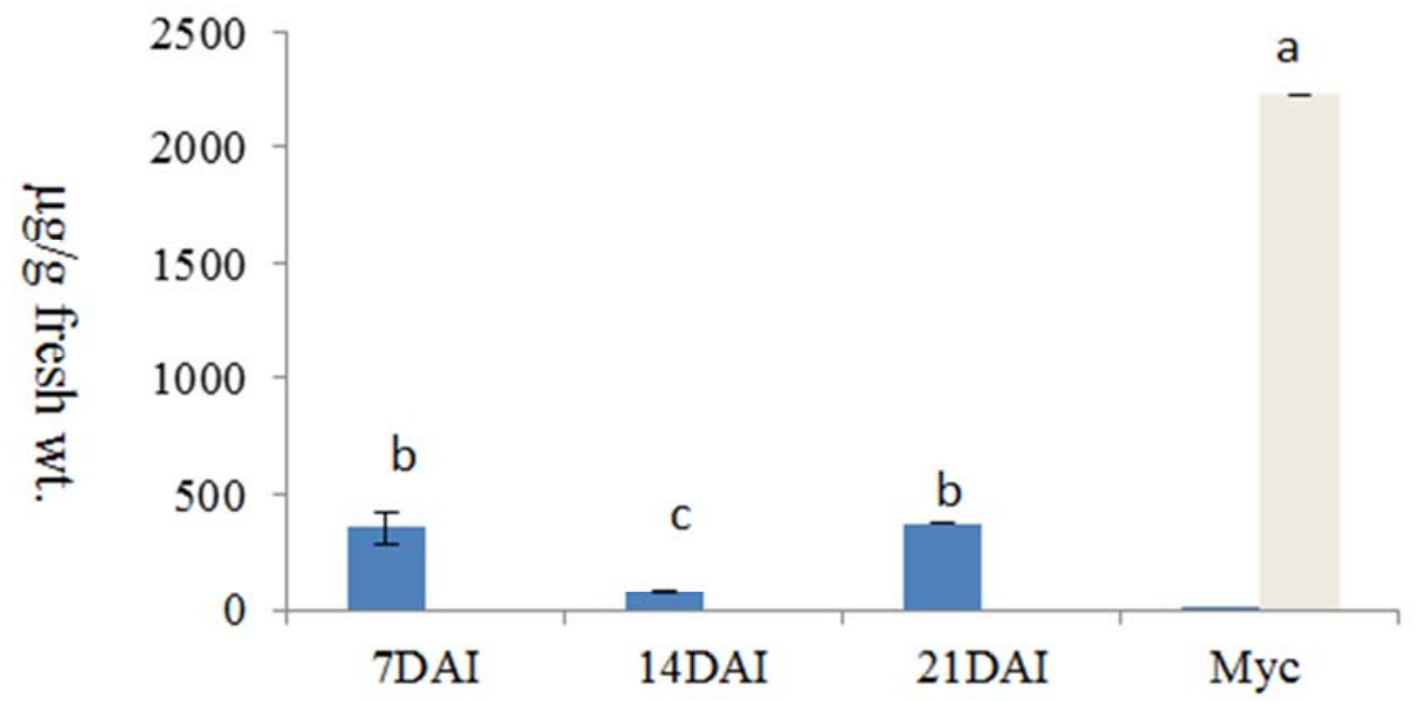

Figure 8. Fusaric acid (FA) quantification in fungal mycelium and from whole plant sample of susceptible variety MR 211 infected with Fusarium proliferatum at different days after inoculation. Myc=mycellium. (Values are means of 3 replications with vertical bar representing standard error).

\subsection{Relationship of Fungal Metabolites at Different Disease Score Levels in Bakanae Diseased Plants}

Amount of FA and MON metabolites isolated from infected susceptible variety MR 211 in relation to bakanae disease development and symptoms expression at different disease score levels are presented in Figure 9 and Figure 10. A polynomial relationship was observed for association of FA 
at different disease score levels, whereas MON was increased in a logarithmic pattern $\left(\mathrm{R}^{2}=0.984\right)$ from disease score level 1 to 5 . Higher amounts of FA was found associated with disease development and symptoms expression at disease score level 1 and 5 (Figure 9). In contrast, MON association in relation to disease development and symptoms expression was observed to be strongly correlated for disease score levels 1 to 5 (Figure 10) in infected plants. The amounts of FB1 and BEA metabolites in relation to disease development in susceptible as well as in resistant variety are presented in Figure 11 and 12 .

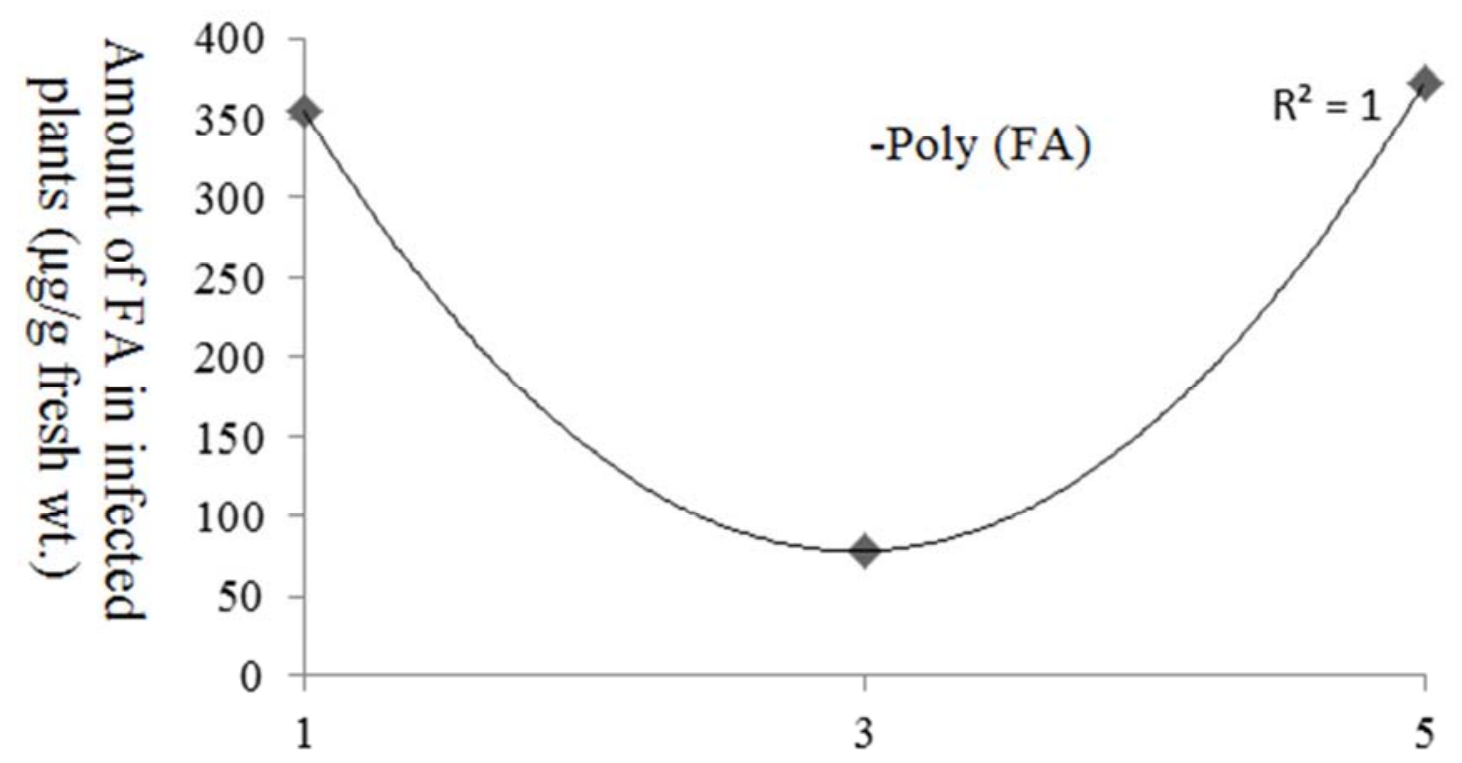

Figure 9. Amount of metabolite FA in relation to bakanae disease development through symptoms expression in infected susceptible variety MR 211 at different disease score levels [( $1=$ Disease score level 1(7 DAI), $3=$ Disease score level 3 (14 DAI), $5=$ Disease score level 5 (21DAI). FA=fusaric acid].

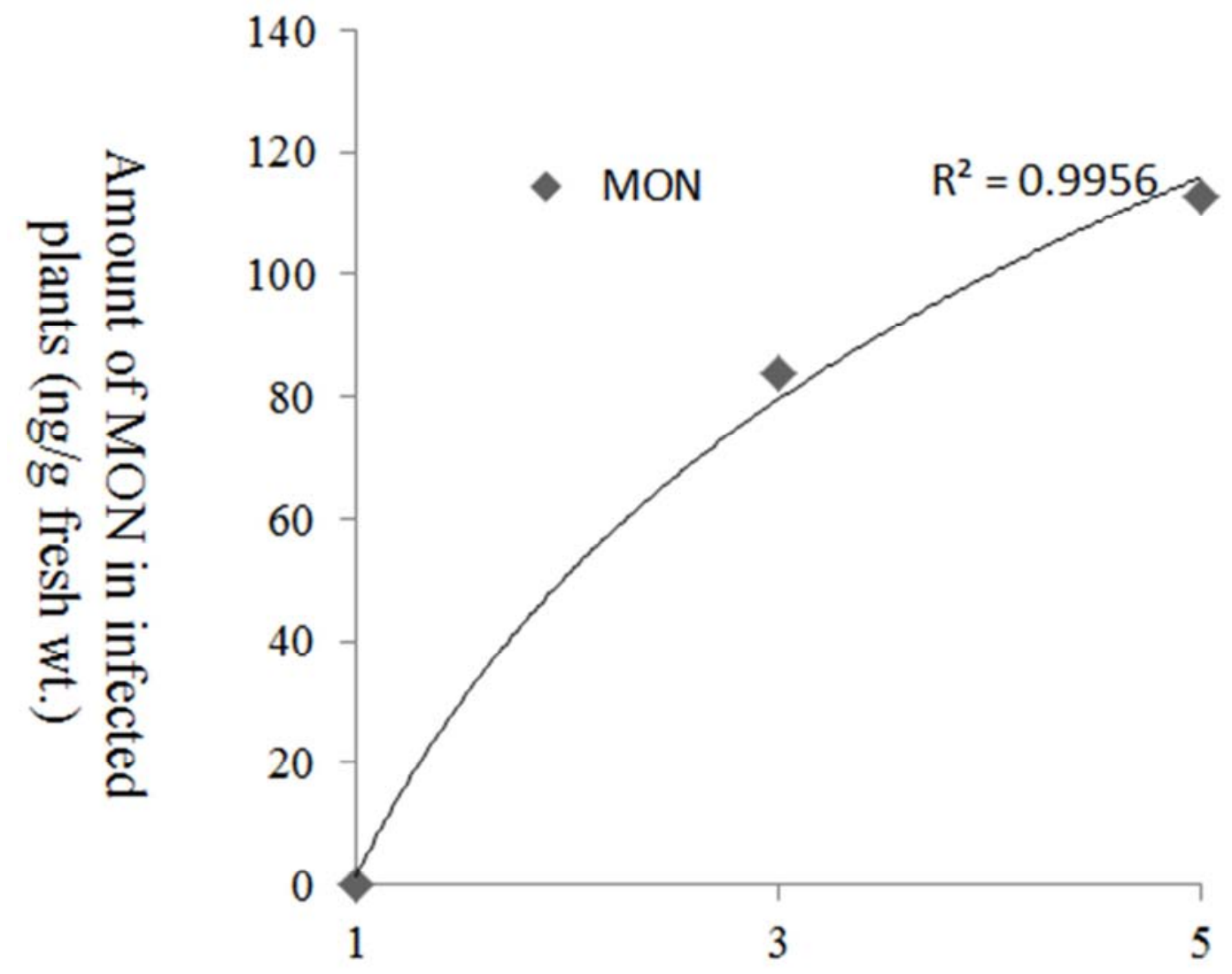

Figure 10. Amount of metabolite MON in relation bakanae to disease development through symptoms expression in infected susceptible variety MR 211 at different disease score levels $[(1=$ Disease score level 1 (7 DAI), $3=$ Disease score level $3(14$ DAI $), 5=$ Disease score level 5 (21DAI). MON = moniliformin]. 


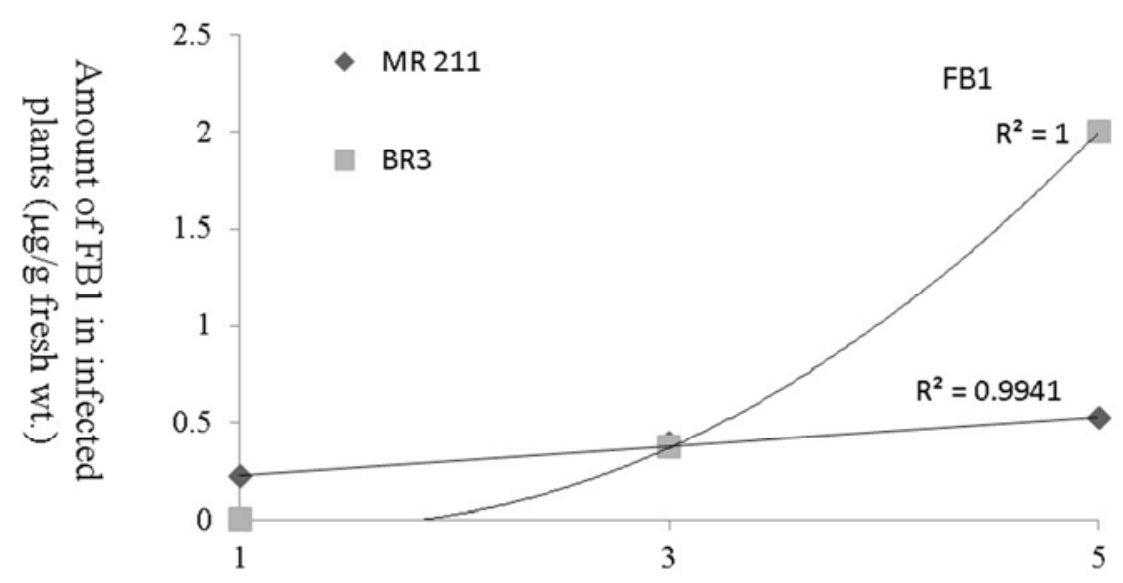

Figure 11. Amount of metabolite FB1 in relation to bakanae disease development through symptoms expression in infected susceptible variety MR 211 and in infected resistant variety BR3 at different disease score levels $[(1=$ Disease score level 1 (7 DAI), $3=$ Disease score level $3(14$ DAI), $5=$ Disease score level $5(21$ DAI). FB1 = fumonisin $]$.

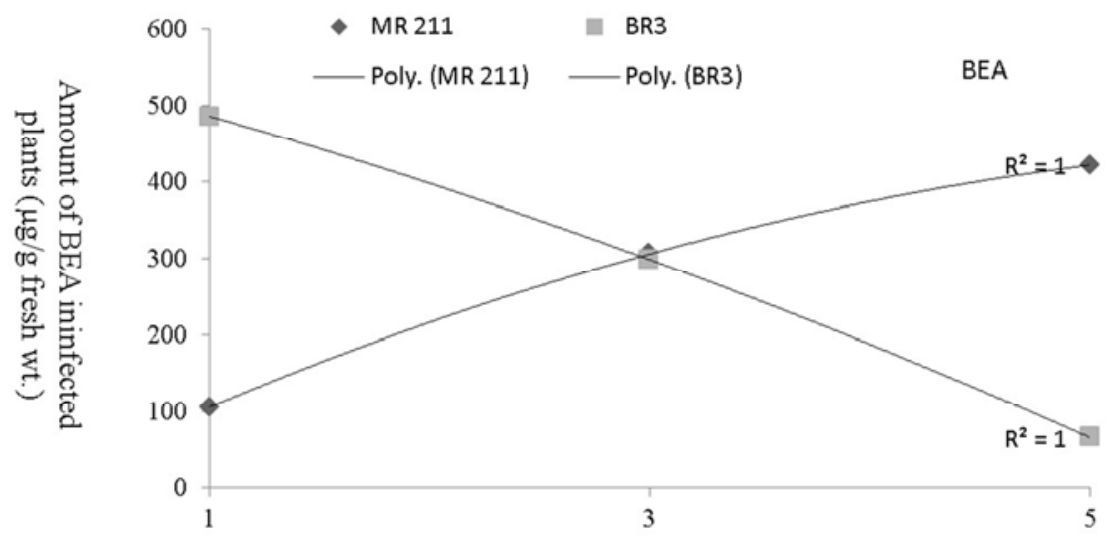

Figure 12. Amount of metabolite BEA in relation to bakanae disease development through symptoms expression in infected susceptible variety MR 211 and in infected resistant variety BR3 at different disease score levels [ $(1=$ Disease score level 1 (7 DAI), $3=$ Disease score level $3(14$ DAI), $5=$ Disease score level $5(21$ DAI). BEA = beauvericin].

\section{Discussion}

A significant amount of MON and FA detection level were found to have strong contribution in bakanae disease symptoms development. These two metabolites had been detected in infected susceptible plants rather than resistant plants. Therefore, it is implied that fungal metabolites possesses role in bakanae disease development along with phytohormonal involvement. Similar conclusion was also reported by Isaac[18] that phytotoxins are host selective and produce symptoms at susceptible varieties. The higher amount of FA produced by $F$. proliferatumat 7 DAI and 21 DAI in infected susceptible variety was established contributing to the stunting and retarded growth of plants. Moniliformin was found to be associated with plant collapses higher amount was detected in later stage of infection in susceptible variety MR 211. These toxin level accumulations in different parts of infected plants are depended by susceptibility of cultivars to pathogen infection and disease development. Similar evidence was supported by Waśkiewicz et al. [19]. MON detection in inoculated resistant variety BR3was hindered and found symptomless of bakanae disease.
Higher amounts of FA were detected in the stem part of short plants at 7 DAI (disease score1) in infected plants of susceptible variety MR 211. This higher concentration of FA indicates an association of FA and stunting of the plants. $\mathrm{Wu}$ et al.[20] reported similar effects of FA on reduction in plant height and in root length. Moreover, Lee and Crill [21] observed that there was a high negative correlation $(\mathrm{r}=$ 0.80 ) between plant height and FA concentration in bakanae diseased susceptible plants. Therefore, it is understood that high levels of FA produced by $F$. proliferatum mycelium might have blocked the transportation of $\mathrm{GA}_{3}$ synthesized from young leaves to internodes of infected susceptible variety MR 211, and thus, plants showed stunting symptom at 7 DAI 1 (disease score 1).

Consequently, increasing FA concentration at 21 DAI (disease score 5), growth rate of infected plants of MR 211 was hampered and finally ceased to death. Desjardins et al. [13] also reported that, release of toxin/s (metabolite/s) by the fungus could be attributed to plant death. Moreover, it has been reported that FA acts synergistically with other toxins produced in the infected plants, and thereby, enhances toxicity of other phytotoxins and plants begin to die [22]. Increased rate of FA accumulation in the leaves and stems 
might have contributed to decrease in photosynthesis rate, chlorophyll content and thereby expressing wilting and necrotic symptoms at $21 \mathrm{DAI}$ (disease score 5). Similar result has been observed by $\mathrm{Wu}$ et al. [20] with the application of FA onto water melon plants. On the other hand, infected resistant BR3 plants showed no bakanae symptoms at this stage. Furthermore, FA was not produced by the $F$. proliferatum in infected plants of BR3 resulting in the plants remaining healthy.

Effect of MON in relation to disease symptoms expression in susceptible variety needs to be evaluated as it was only detected in infected susceptible variety MR 211 and was not present in resistant inoculated variety BR3. Effect of MON on crown rot and root necrosis in bakanae diseased plants at 21 DAI (disease score 5) should be investigated as highest level of MON was isolated in this stage. Feng et al. [23] also reported that there might be an association of metabolites produced by Fusarium species with plants root rot. In earlier, researchers also suspected that there might be a positive relationship of bakanae disease development and MON produced by the strains of G. fujikuroi [24].

Some yellowing of leaves in infected BR3 plants at the late tillering stage might be due to the effect of higher amount of FB1 accumulation in leaves produced by the fungus. Igarashi et al.[25]also reported that FB1 caused yellowing of leaves in Arabidopsis mature leaves after three to four days of inoculation by causing Programmed cell death. Moreover, FB1 treated leaves developed lesions with characteristics reminiscent of hypersensitive response, including the generation of reactive oxygen species, deposition of phenolic compounds and callose, accumulation of camalexin, and expression changes of defense-related genes [26]. Other researcher reported that FB1 was implicated as an aetiological agent in disease development as the FB1 treated jimsonweed plants produced similar response to symptoms [27]. Moreover, a relationship was established between fumonisins and high levels of virulence on maize seedlings [28]. On contrary, Stone et al. [26] recommended that although cell death is a central feature of both compatible and incompatible plant-pathogen interactions, its respective roles in resistance and susceptibility are largely unknown.

Effect of BEA was not found directly on bakanae disease symptoms expression. BEA might have connection with disease development and symptoms expression in association with other metabolites. BEA has been found to inhibit root transpiration in susceptible variety of maize [29]. High accumulation of BEA might be reflected indirectly on disease symptoms expression in susceptible variety MR 211, with enhancement in cell death at 21DAI (disease score 5), whereas it was not reflected in infected resistant variety BR3. Wakulinski et al. [30] supported this evidence that BEA caused a decreased in cell viability and DNA fragmentation resulting in cell death in wheat seedling. In fact, the effect of BEA on cell death has been found in association with increased reactive oxygen species that correlated with increased $\mathrm{H}_{2} \mathrm{O}_{2}$ in infected plants [31].

Relationship studies between symptoms expression and involvement of metabolites produced by $F$. proliferatum in infected plants in susceptible variety, revealed that FA was associated with disease symptoms expression in disease score 1 (plant stunted with chlorotic leaves) and 5 (leaf and stem browning with elongated internodes or dead plant). Lower amount of FA association was observed in disease score 3 (abnormal elongated internodes with chlorotic or brownish leaves), and it was vice versa in disease score 1 that resulted in stunting of plants. A polynomial relationship for FA and logarithmic relationship for MON was found in association with different disease score levels. However, higher amounts of FA and MON produced by $F$. proliferatum in infected plants at disease score 5 , might be responsible for a decrease in the amount of $\mathrm{GA}_{3}$, thereby decreasing root length and ceased growth, plant death, and necrosis of roots in susceptible variety MR 211. It is assumed that FA and MON act synergistically, and thereby expressing different symptoms depending on the combination of FA and MON at different disease score levels. This similar explanation was also supported by other researchers that stem and crown rot of asparagus disease was associated with presence of higher level of mycotoxins produced by the causal fungus [19].

From this study, it can be concluded that there is a relationship between fungal metabolites production in diseased plants and symptoms expression. Among the four metabolites, higher amount of FA is associated with plant stunting/ceasing growth, along with leaf chlorosis at disease score level 1and 5. Higher amount of MON production at disease score levels 1, 3 and 5 are related with root growth retardation and root necrosis. Further investigations by applying synthetic FA and MON as seed treatment are needed for more clarification on the role of FA and MON in bakanae disease development and symptoms expression.

\section{Acknowledgements}

The authors wish to express their sincere thanks and appreciations to Universiti Putra Malaysia (UPM) for the research grant administered through RUGS (01-04-10-1040RU), the Bangladesh Agricultural Research Council (BARC) through a Doctoral fellowship and the Institute of Tropical Agriculture and the Faculty of Agriculture, Universiti Putra Malaysia for providing the research facilities.

\section{References}

[1] Quazi, S. A. J., Meon, S., Jaafar, H., Ahmad, Z. A. B. M. (2013) Characterization of Fusarium proliferatum through species specific primers and its virulence on rice seeds. International Journal of Agriculture and Biology, 15: 649-56.

[2] Quazi, S. A. J., Meon, S., Jaafar, H. \& Ahmad Z. A. B. M. (2015) The role of phytohormones in relation to bakanae disease development and symptoms expression. Physiological and Molecular Plant Pathology, 90: 27-38.

[3] Leslie, J. F., Anderson, L. L., Bowden, R. L. and Lee, Y.-W. (2007) Inter-and intra-specific genetic variation in Fusarium. International Journal of Food and Microbiology, 119: 25-32. 
[4] Syahputra, B. S. A., Sinniah, U. R., Rastan, S. O. S., and Ismail, M. R. (2013) Changes in gibberellic acid $\left(\mathrm{GA}_{3}\right)$ content in Oryza sativa due to paclobutrazol treatment. Journal of Food and Pharmaceutical Sciences, 1: 14-17.

[5] Proctor, R. H., Desjardins, A. E., and Moretti, A. (2010) Biological and chemical complexity of Fusarium proliferatum. In Strange, R. N. and Gullino. M. L. Ed.,The Role of Plant Pathology in Food Safety and Food Security, Plant Pathology in the 21stCentury(volume 3). Netherlands: Springer, 97-111

[6] Cruz, A., Marin, P., González-Jaén, M. T., Aguilar, K. G. and Cumagun, C. J. R. (2013) Phylogenetic analysis, fumonisin production and pathogenicity of Fusarium fujikuroi strains isolated from rice in the Philippines. Journal of Science Food andAgriculture 93: 3032-3039.

[7] Amatulli, M. T., Spadaro, D., Gullino, M. L., Garibaldi, A. (2010) Molecular identification of Fusarium spp. associated with bakanae disease of rice in Italy and assessment of their pathogenicity. Plant Pathology 59: 839-844.

[8] Li, C., Wu, Y-L., Yang, T. and Huang-Fu, W-G. (2012) Rapid determination of fumonisins B1 and B2 in corn by liquid chromatography-tandem mass spectrometry with ultrasonic extraction. Journal of Chromatographic Science, 50: 57-63.

[9] Parmar, P., Oza, V. P. and Subramanian, R. B. (2010) Optimization of fusaric acid production by Fusarium oxysporum f. sp. lycopersici using response surface methodology. Indian Journal of Science and Technology,3(4): 411-416.

[10] Šrobárová, A., Eged, S., Da Silv, a J. T., Ritieni, A. and Santini, A. (2009) The use of Bacillus subtilis for screening fusaric acid production by Fusarium spp., Czech Journal of Food Sciences, 27 (3): 203-209.

[11] Liu, F-M., Chen, P-C., Fu, Y-M. and Shih, D. Y-C. (2005) Determination of fumonisin B1and B2 in corn products. Journal of Food and DrugAnalysis, 13(3): 273-278.

[12] Desjardins, A. E. and Hohn, T. M. (1997) Mycotoxins in plant pathogenesis MolePlant-Microbe Interaction, 10(2): 147-152.

[13] Desjardins, A. E., Manandhar, H. K., Plattner, R. D., Anandhar, G. G., Poling, S. M. and Maragos, C. M. (2000) Fusarium species from Nepalese rice and production of mycotoxins and gibberellic acid by selected species. Applied and Environmental Microbiology, 66(3): 1020-1025.

[14] Kovačić, S., Pepeljnjak, S., Petrinec, Z. and Mklarić, M. S. (2009) Fumonisin B1 Neurotoxicity. In Young carp (Cyprinus Carpio L.). Archives of Industrial Hygiene and Toxicol, 60:419-426.

[15] Munimbazi, C. and Bullerman, L. B. (2001) Chromatographic method for the determination of the mycotoxin moniliformin in corn. In Trucksess, M. W. and Pohland, A. E. Ed., Methods in Molecular Biology. Mycotox Protoc 157 Totowa, Newzealand: Humana Press. 131-145.

[16] Kostecki, M., Wisniewska, H., Perrone, G., Ritieni, A., Jerzy, P. G., Chelkowski, J. and Logrieco, A. (1999) The effects of cereal substrate and temperature on production of beauvericin, moniliformin and fusaproliferin by Fusarium subglutinans ITEM-1434. Food Additives and Contaminants: Chemistry, Analysis, Control, Exposure \& amp; Risk Assessment, 16(9): 361-365 (available online on: http://dx.doi.org/10.1080/026520399283849)
[17] Eged, S. (2005) Thin-layer chromatography.an appropriate method for fusaric acid estimation. BiologiaBratislava, 60(1): 104.

[18] Issac., S. (1998) Some fungi produce toxins that affect the physiological functioning of higher plants: what is the role of these compounds in the development of plant disease? Mycologist, 12(3): 124-125.

[19] Waśkiewicz A, Irzykowska L, Bocianowski J, Karolewski Z, Kostecki1 M, Weber Z, and Goliński1 P (2010) Occurrence of Fusarium Fungi and Mycotoxins in Marketable Asparagus Spears. Polish Journal of Environmental Studies, 19(1): 219225.

[20] Wu, H-S., Bao, W., Liu, D-Y., Ling, N., Ying, R-R., Raza, W. and Shen, Q-R. (2008). Effect of fusaric acid on biomass and photosynthesis of watermelon seedlings leaves. Caryologia. 61(3): 258-268.

[21] Lee, Y. H. and Crill, J. P. (1989) Role of gibberellic acid and fusaric acid in rice plant inoculated with Gibberella fujikuroi (Swada) ito and kimura. Korean Journal of Plant Pathology, 5(2): 126-130.

[22] Bacon, C. W., Porter, J. K., Norred, W. P., and Leslie, J. F. (1996). Production of fusaric acid by Fusarium species. Applied and Environmental Microbiology, 62(11): 4039-4043.

[23] Feng, J., Hwang, R., Chang, K. F., Hwang, S. F., Strelkov, S. E., Gossen, B. D., Conner, R. L. and Turnbull, G. D. (2010) Genetic variation in Fusariumavenaceum causing root rot on field pea. Plant Pathology, 59: 845-852.

[24] Marasas, W. F. 0., Thiel, P. G., Rabie, C. J., Nelson, P. E., and Toussoun, T. A. (1986) Moniliformin production in Fusarium section Liseola. Mycologia, 78: 242-247.

[25] Igarashi, D., Bethke, G., Xu, Y., Tsuda, K., Glazebrook, J., and Katagiri, F. (2013) Pattern-Triggered Immunity Suppresses Programmed Cell Death Triggered by Fumonisin B1. PLoS ONE 8(4): e60769. doi:10.1371/journal.pone.0060769.

[26] Stone J. M., Heard, J. E., Asai, T., Ausubel F. M. (2000) Simulation of fungal-mediated cell death by fumonisin B1 and selection of fumonisin B1-resistant (fbr) Arabidopsis mutants. Plant Cell, 12: 1811-1822.

[27] Abbas, H. K., Boyette, C. D., Hoagland, R. E., and Vesonder, R. E. (1991) BioherbicidalpotentialofFusarium moniliforme and its phytotoxin, fumonisin B1. Weed Science, 139, 673-677.

[28] Desjardins, A. E., Plattner, R. D., Nelsen, T. C., and Leslie, J. F. (1995) Genetic analysis of fumonisin production and virulence of Gibberella fujikuroi mating population A (Fusarium mOllilifomle) on maize (Zea mays) seedlings. Applied Environmental and Microbiology, 61: 79-86.

[29] Pavlovkin, J., Mistríková, I., Luxová, M. and Mistrík, I. (2006) Effects of beauvericin on root cell transmembrane electric potential, electrolyte leakage and respiration of maize roots with different susceptibility to Fusarium. Plant, Soil and Environment 52(11): 492-498.

[30] Wakulinski, W. (1989). Phytotoxicity of the secondary metabolites of fungi causing wheat head fusariosis (head blight). Acta Physiologia Plantarum,11: 301-306.

[31] Paciolla, C., Dipierro, N., Mule, G., Logrieco, A. and Dipierro, S. (2004) The mycotoxins beauvericin and T-2 induce cell death and alteration to the ascorbate metabolism in tomato protoplasts. Physiological and Molecular Plant Pathology, 65: 49-56. 